\title{
Surgery for temporo-mesial epilepsy: what best?
}

\author{
Marc Sindou
}

Received: 25 November 2010 / Accepted: 25 November 2010/Published online: 9 December 2010

(C) Springer-Verlag 2010

This issue of Acta Neurochirurgica devotes significant attention to a crucial problem in epilepsy surgery-what is the posterior extent of mesial temporal resection for effective control of seizures? Up till now, this problem had not been precisely investigated as the sole variable factor. With a two-part article entitled "Randomized controlled trial of $2.5 \mathrm{~cm}$ versus $3.5 \mathrm{~cm}$ mesial temporal resection in temporal lobe epilepsy", one of Europe's leading teams in the field provides an answer.

Presentation of the work as two separate papers - the first being "Intent-to-treat analysis" and the second being "Volumetric resection and subgroups analysis"- is somewhat difficult to follow. This was done for statistical reasons, as the study was launched with randomization of the intended extent of resection of the hippocampal $(\mathrm{HC})+$ parahippocampal (pHC) structures-2.5 versus $3.5 \mathrm{~cm}$ posteriorly.

Extent of resection was determined following opening of the temporal horn, by using a millimetric paper strip. This was measured from the anterior tip of the temporal horn posteriorly and the paper strip was placed on the hippocampal head along its longitudinal axis. Although a theoretically precise method under the operative microscope, post-operative MRI demonstrated that "true extent varied considerably from estimation of the $\mathrm{HC} / \mathrm{pHC}$ resection at surgery". These discrepancies were however considered not redhibitory by the authors as the mean true resection volumes were found to be significantly different between the $2.5-$ and the $3.5-\mathrm{cm}$ resection groups $(72.86 \%$

\section{Sindou $(\bowtie)$}

Department of Neurosurgery, University Claude-Bernard of Lyon I,

Hôpital Neurologique P. Wertheimer, GHE,

59 Bd Pinel,

69003 Lyon, France

e-mail: marc.sindou@chu-lyon.fr of the initial volume in the $2.5 \mathrm{~cm}$ group versus $83.44 \%$ in the 3.5 group). The study also showed that there was no significant difference in seizure outcome at 1 year between the two groups. Comparing the $2.5-$ and $3.5-\mathrm{cm}$ resection groups, Engel's class I outcome rates were $74 \%$ and $72.8 \%$, respectively ( $p=0.843)$. The authors conclude that: "once an average of $60-69 \%$ of mesial temporal lobe volume is resected, an increasing extent of further mesial resection does not lead to an increasing frequency of seizure freedoms".

However, when looking at Table 1 in part 2 of the article, it appears that for patients having had selective amygdalo-hippocampectomy (125 patients), Engel's class I outcome rate was better for $3.5-\mathrm{cm}$ resection $(72.3 \%)$ than for $2.5-\mathrm{cm}$ resection $(61.7 \%)$. But the difference was not found to be statistically significant $(p=0.207)$.

A limitation of the work is that there was a certain degree of heterogeneity within the series. For example, there were important differences in the method of temporal resections among the three centers enrolled - one preferred selective amygdalo-hippocampectomy (SAH), while another favored two-third temporal lobectomy. However, if only the extent of temporo-mesial resection is considered as the sole variable factor, the authors' conclusion of : "Extending the length at $3.5 \mathrm{~cm}$ instead of $2.5 \mathrm{~cm}$, does not play a significant role in seizure-free outcome", can be taken for granted. This is especially important as posterior limitation of resection is crucial for prevention of memory deficits.

Another important issue of the authors' study is that Engel's class I outcome was found to be higher in patients who had anterior or two-third temporal lobectomy with their mesial resections $(83.8 \%$ in their 74 patients) than in those who had selective amygdalo-hippocampectomy (67.2\% in their 125 patients). But this difference was not statistically analyzed. Although the authors note that "study 
design did not randomize patients for surgery types", difference in outcome should not be neglected, especially in patients who do not have evidence of mesio-temporal sclerosis on MRI.

In our experience, resection of mesial structuresadditionally including the pole-achieves better results than SAH alone, even in pure temporo-mesial epilepsy (TME). In a recent published report on 100 patients operated on for TME, our Engel's class I outcome was $85 \%$, with $74 \%$ in class Ia [3].

Presurgical chronic depth electrode recordings using the Talairach' stereo-electro-encephalography method showed us that in a majority of patients with TME explored, the pole was implicated early in the onset of epilepsy, i.e., approximately at the same time as the mesial structures themselves [1].

Looking at anatomy, this is not surprising given that the pole, at least its medial aspect, is in continuity with the entorhinal cortex of the uncus as well as with the parahippocampal gyrus. Furthermore, regarding its histological organization, the tempora pole contains meso- cortex, which represents a gradual transition from the "primitive" allocortex of the strictly located mesial temporal structures to the "recent" isocortex of the lateral temporal lobe [2].

\section{Conflicts of interest None.}

\section{References}

1. Guenot M, Isnard J, Ryvlin P, Fischer C, Ostrowsky K, Mauguiere F, Sindou M (2002) Neurophysiological monitoring for epilepsy surgery: the Talairach SEEG method. Stereotact Funct Neurosurg 73:84-87

2. Sindou M, Guenot M (2003) Surgical anatomy of the temporal lobe for epilepsy surgery. In: Pickard JD et al (eds) Advances and technical standards in neurosurgery, Vol 28. Springer, Wien, pp 315-343

3. Sindou M, Guenot M, Isnard J, Ryvlin P, Fischer C, Mauguiere F (2006) Temporo-mesial epilepsy surgery: outcome and complications in 100 consecutive adult patients. Acta Neurochir Wien $148: 39-45$ 